\title{
Oxygen Nonstoichiometry and Valence State of Manganese in $\mathrm{La}_{1-x} \mathrm{Ca}_{x} \mathrm{MnO}_{3+\delta}$
}

Sabrina A. Heuer ${ }^{1,2}$, Roland Schierholz ${ }^{* 1}$, Evgeny V. Alekseev ${ }^{1}$, Lars Peters ${ }^{3}$, David N. Mueller ${ }^{4}$, Tomáš Duchoň ${ }^{4}$, Vaibhav Vibhu ${ }^{1}$, Hermann Tempel ${ }^{1}$, Lambertus G. J. de Haart ${ }^{1}$, Hans Kungl ${ }^{1}$ and Rüdiger-A. Eichel ${ }^{1,2}$

${ }^{1}$ Forschungszentrum Jülich GmbH, Institute of Energy and Climate Research (IEK-9), Wilhelm-Johnen-Straße, DE-52425 Jülich, Germany

${ }^{2}$ RWTH Aachen University, Institute of Physical Chemistry, Landoltweg 2, DE-52074 Aachen, Germany

${ }^{3}$ RWTH Aachen University, Institute of Crystallography, Jägerstraße 17-19, DE-52066 Aachen, Germany

${ }^{4}$ Forschungszentrum Jülich GmbH, Peter Gruenberg Institute (PGI-6), Wilhelm-Johnen-Straße, DE-52425 Jülich, Germany

KEYWORDS: Oxygen-nonstoichiometry, Defect chemistry, $\mathrm{LaMnO}_{3}$, Thermogravimetry, XANES. 

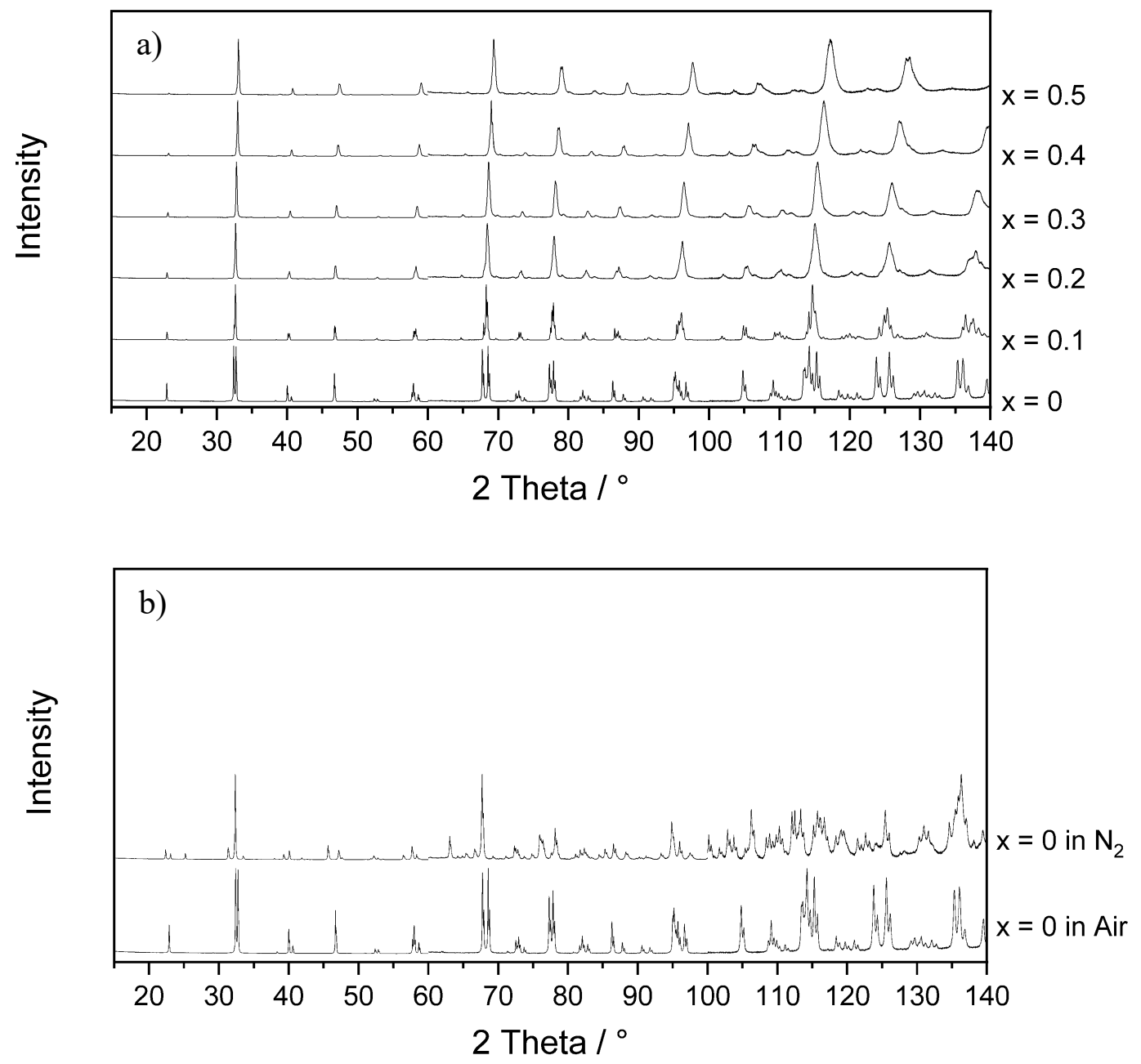

Figure S1: (a) PXRD patterns of Ca-substituted $\mathrm{La}_{1-\mathrm{x}} \mathrm{Ca}_{\mathrm{x}} \mathrm{MnO}_{3+\delta}$ synthesized in air, measured in three separate ranges and (b) PXRD patterns of $\mathrm{LaMnO}_{3}$ and $\mathrm{La}_{0.9} \mathrm{Ca}_{0.1} \mathrm{MnO}_{3+\delta}$ prepared in air and additionally tempered in $\mathrm{N}_{2}$.

Table S1: Determination of structure, lattice parameters $(a, b$ and $c)$ and unit cell volume of air-synthesized $\mathrm{La}_{1-x} \mathrm{Ca}_{x} \mathrm{MnO}_{3+\delta}$

\begin{tabular}{|c|c|c|c|c|c|c|c|}
\hline $\mathrm{x}$ & Structure $^{*}$ & $a(\AA)$ & $b(\AA)$ & $b / \sqrt{2}(\AA)$ & $c(\AA)$ & $\alpha\left(^{\circ}\right)$ & $\mathrm{V}_{\text {unit }}\left(\AA^{3}\right)$ \\
\hline \multirow[t]{2}{*}{0 (air) } & $\mathrm{R}_{\text {trigonal }}$ & $5.47310(2)$ & $5.47310(2)$ & & $5.47310(2)$ & 60.69 & \\
\hline & $\mathrm{R}_{\text {hexagonal }}$ & $5.52961(2)$ & $5.52961(2)$ & & $13.33640(5)$ & & $58.8582(4)$ \\
\hline $0\left(\mathrm{~N}_{2}\right)$ & $\mathrm{O}^{\prime}$ & $5.70438(4)$ & $7.70664(6)$ & $5.44942(4)$ & $5.53779(5)$ & & $60.8625(9)$ \\
\hline 0.1 & $\mathrm{O}$ & $5.48071(3)$ & $7.75459(4)$ & $5.48332(3)$ & $5.51694(2)$ & & $58.6183(5)$ \\
\hline 0.2 & $\mathrm{O}$ & $5.47266(7)$ & $7.74137(9)$ & $5.47398(6)$ & $5.50091(6)$ & & $58.263(2)$ \\
\hline 0.3 & $\mathrm{O}$ & $5.46811(7)$ & 7.72917(9) & $5.46535(7)$ & $5.48332(6)$ & & $57.937(2)$ \\
\hline 0.4 & $\mathrm{O}$ & $5.44308(6)$ & 7.68524(7) & $5.43428(5)$ & $5.45876(5)$ & & $57.087(1)$ \\
\hline 0.5 & $\mathrm{O}$ & $5.41906(7)$ & $7.64203(8)$ & $5.40373(6)$ & $5.43157(7)$ & & $56.234(2)$ \\
\hline
\end{tabular}




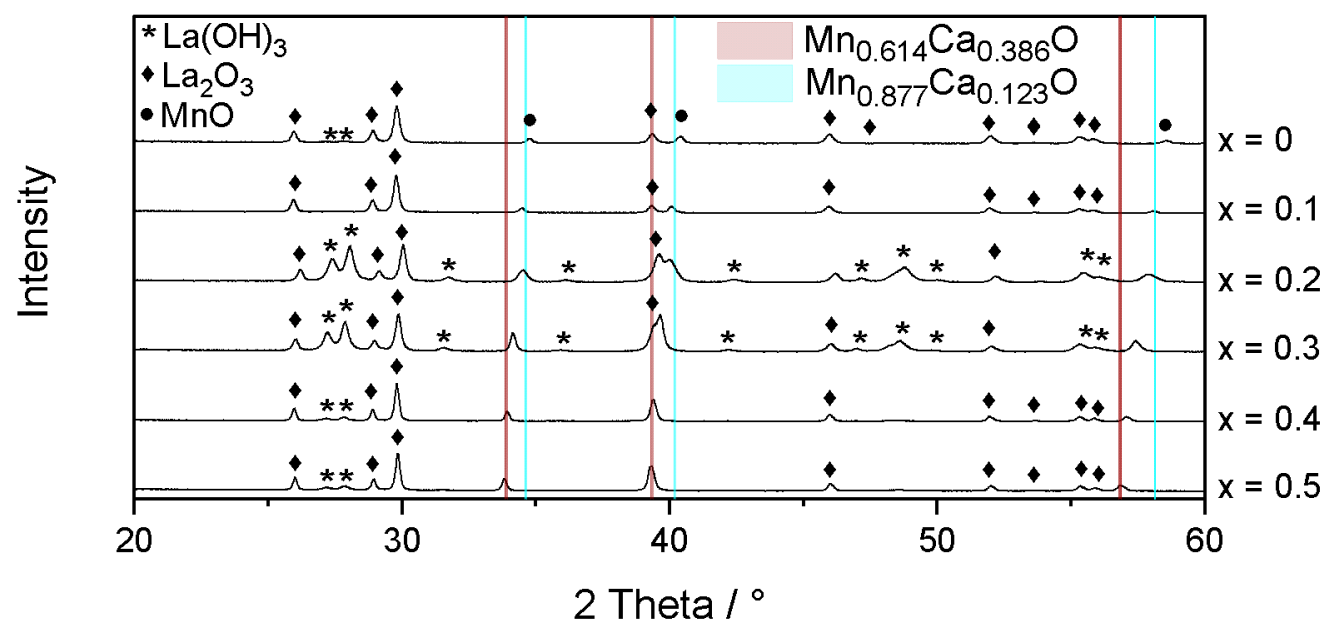

Figure S2: PXRD measurements of the decomposition products of the $\mathrm{La}_{1-x} \mathrm{Ca}_{x} \mathrm{MnO}_{3+\delta}$ series after reduction in $4 \% \mathrm{Ar} / \mathrm{H}_{2}$-atmosphere upon thermogravimetric analysis.

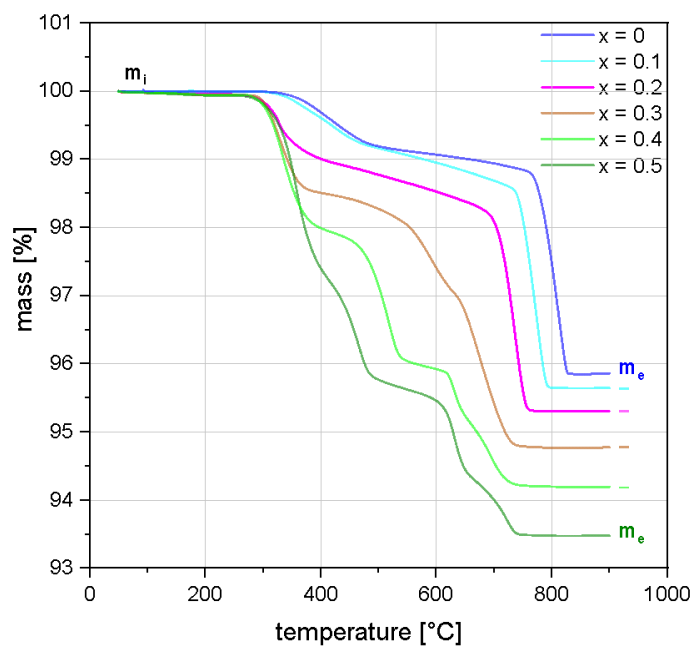

Figure S3: Reduction of the air-synthesized $\mathrm{La}_{1-\mathrm{x}} \mathrm{Ca}_{\mathrm{x}} \mathrm{MnO}_{3+\delta}$ $(\mathrm{x}=0-0.5)$ in $\mathrm{Ar} / 4 \% \mathrm{H}_{2}$ atmosphere. The samples are heated to $900^{\circ} \mathrm{C}$ in a reducing atmosphere, where they decompose into their specific products and oxygen. Oxygen release is assumed to cause the obtained mass difference used to determine the $\delta$-value of the samples La${ }_{x} \mathrm{Ca}_{x} \mathrm{MnO}_{3+\delta}$. 


$$
\Delta \boldsymbol{\delta}_{T G A}=-\frac{M_{L C M O}+\delta \cdot M_{O}-\frac{m_{e}}{m_{i}} \cdot\left(M_{L C M O}+\delta \cdot M_{O}\right)}{M_{O}} \quad \text { Equation S1 }
$$

Formula used for the calculation of changes in $\delta$-values along with temperature, atmosphere and Ca-content (Table S2). $M_{\mathrm{LCMO}}$ is the molar mass of $\mathrm{La}_{1-\mathrm{x}} \mathrm{Ca}_{\mathrm{x}} \mathrm{MnO}_{3.00}$ and $M_{\mathrm{O}}$ is the molar mass of oxygen. The $\delta$-value represents the initial oxygen non-stoichiometry of the sample. The mass ratio $\frac{m_{e}}{m_{i}}$ is the initial sample mass divided by the end mass. The end mass is not specific, it can be chosen as desired. In this work, two mass areas have been chosen as end masses. They are named either $m_{950 C_{-} 2 n d}$ or $m_{\text {end }}$. The respective areas can be found in Figure 5 .

Table S2: Calculation of the of the $\delta$-value changes $\Delta \delta$ from the TGA-experiments shown in Figure $4(\mathrm{i})$ at $950{ }^{\circ} \mathrm{C}$ during the second cycle for Figure 7 (a) (ii) and at RT after the two cycles for Figure 7 (b).

\begin{tabular}{|c|c|c|c|c|c|c|c|c|c|}
\hline \multicolumn{10}{|l|}{$\mathrm{O}_{2}$} \\
\hline$x$ & $\delta_{\text {initial }}$ & $M_{\mathrm{LCMO}}(\delta=0)$ & $3+\delta_{\text {initial }}$ & {$\left[m_{950 \_2 n d} / m_{\mathrm{i}}\right]$} & $\Delta \delta_{950 \_2 n d}$ & $3+\delta_{950 \_2 \text { nd }}$ & {$\left[m_{\mathrm{e} \_2 n d} / m_{\mathrm{i}}\right]$} & $\Delta \delta_{\text {end }}$ & $3+\delta_{\text {end }}$ \\
\hline 0 & 0.137 & 241.845 & 3.137 & 0.9980 & -0.031 & 3.106 & 1.0006 & 0.008 & 3.145 \\
\hline 0.1 & 0.088 & 231.962 & 3.088 & 0.9976 & -0.035 & 3.053 & 1.0002 & 0.003 & 3.091 \\
\hline 0.2 & 0.047 & 222.079 & 3.047 & 0.9982 & -0.026 & 3.021 & 1.0001 & 0.001 & 3.048 \\
\hline 0.3 & 0.011 & 212.195 & 3.011 & 0.9996 & -0.006 & 3.005 & 1.0002 & 0.002 & 3.013 \\
\hline 0.4 & 0.01 & 202.312 & 3.010 & 0.9996 & -0.006 & 3.004 & 0.9996 & -0.005 & 3.005 \\
\hline 0.5 & 0.011 & 192.429 & 3.011 & 0.9994 & -0.007 & 3.004 & 0.9995 & -0.007 & 3.004 \\
\hline \multicolumn{10}{|l|}{ air } \\
\hline$x$ & $\delta_{\text {initial }}$ & $M_{\mathrm{LCMO}}(\delta=0)$ & $3+\delta_{\text {initial }}$ & {$\left[m_{950 \_2 n d} / m_{\mathrm{i}}\right]$} & $\Delta \delta_{950 \_2 n d}$ & $3+\delta_{950 \_2 n d}$ & {$\left[m_{\mathrm{e} \_2 n d} / m_{\mathrm{i}}\right]$} & $\Delta \delta_{\text {end }}$ & $3+\delta_{\text {end }}$ \\
\hline 0 & 0.137 & 241.845 & 3.137 & 0.9967 & -0.050 & 3.087 & 0.9994 & -0.009 & 3.128 \\
\hline 0.1 & 0.088 & 231.962 & 3.088 & 0.9966 & -0.050 & 3.038 & 0.9991 & -0.013 & 3.075 \\
\hline 0.2 & 0.047 & 222.079 & 3.047 & 0.9976 & -0.033 & 3.014 & 0.9993 & -0.010 & 3.037 \\
\hline 0.3 & 0.011 & 212.195 & 3.011 & 0.9995 & -0.007 & 3.004 & 0.9997 & -0.003 & 3.008 \\
\hline 0.4 & 0.01 & 202.312 & 3.010 & 0.9995 & -0.006 & 3.004 & 0.9996 & -0.005 & 3.005 \\
\hline 0.5 & 0.011 & 192.429 & 3.011 & 0.9992 & -0.009 & 3.002 & 0.9993 & -0.008 & 3.003 \\
\hline \multicolumn{10}{|l|}{$\mathrm{N}_{2}$} \\
\hline$x$ & $\delta_{\text {initial }}$ & $M_{\mathrm{LCMO}}(\delta=0)$ & $3+\delta_{\text {initial }}$ & {$\left[m_{950 \_2 n d} / m_{\mathrm{i}}\right]$} & $\Delta \delta_{950 \_2 n d}$ & $3+\delta_{950 \_2 \text { nd }}$ & {$\left[m_{\mathrm{e} \_2 n d} / m_{\mathrm{i}}\right]$} & $\Delta \delta_{\text {end }}$ & $3+\delta_{\text {end }}$ \\
\hline 0 & 0.137 & 241.845 & 3.137 & 0.9916 & -0.129 & 3.008 & 0.9920 & -0.122 & 3.015 \\
\hline 0.1 & 0.088 & 231.962 & 3.088 & 0.9935 & -0.095 & 2.993 & 0.9939 & -0.089 & 2.999 \\
\hline 0.2 & 0.047 & 222.079 & 3.047 & 0.9966 & -0.047 & 3.000 & 0.9967 & -0.045 & 3.002 \\
\hline 0.3 & 0.011 & 212.195 & 3.011 & 0.9993 & -0.010 & 3.001 & 0.9994 & -0.008 & 3.003 \\
\hline 0.4 & 0.01 & 202.312 & 3.010 & 0.9994 & -0.007 & 3.003 & 0.9995 & -0.007 & 3.003 \\
\hline 0.5 & 0.011 & 192.429 & 3.011 & 0.9990 & -0.012 & 2.999 & 0.9992 & -0.010 & 3.001 \\
\hline
\end{tabular}

